\title{
A STUDY OF FUNCTIONAL, CLINICAL AND RADIOLOGICAL OUTCOME OF FRACTURE SHAFT OF HUMERUS MID AND DISTAL THIRD MANAGED BY MIPPO TECHNIQUE
}

\author{
Subramanian Karunanithi ${ }^{1}$, Srinivasan Anbu ${ }^{2}$, Mathivanan Palaniappan ${ }^{3}$, Kalyanasundaram Kolundan ${ }^{4}$, Kishore Kannan ${ }^{5}$, \\ Ramesh Prabu Ganesan 6 \\ ${ }^{1}$ Assistant Professor, Department of Orthopaedics, KAPV Govt. Medical College/MGM Govt. Hospital, Tiruchirapalli. \\ ${ }^{2}$ Associate Professor, Department of Orthopaedics, KAPV Govt. Medical College/MGM Govt. Hospital, Tiruchirapalli. \\ 3 Professor, Department of Orthopaedics, KAPV Govt. Medical College/MGM Govt. Hospital, Tiruchirapalli. \\ ${ }^{4}$ Associate Professor, Department of Orthopaedics, KAPV Govt. Medical College/MGM Govt. Hospital, Tiruchirapalli. \\ ${ }^{5}$ Assistant Professor, Department of Orthopaedics, KAPV Govt. Medical College/MGM Govt. Hospital, Tiruchirapalli. \\ ${ }^{6}$ Assistant Professor, Department of Orthopaedics, KAPV Govt. Medical College/MGM Govt. Hospital, Tiruchirapalli.
}

\section{ABSTRACT}

\section{BACKGROUND}

Minimally invasive percutaneous plate osteosynthesis (MIPPO) technique through the anterior approach has been widely used to treat humeral shaft fractures in recent years, because of its technical advantages and satisfactory clinical outcomes.

\section{OBJECTIVES}

- To observe the anatomical relationship between the radial nerve and plate during supination and pronation of forearm, so as to determine which position of forearm is safest for radial nerve in distal humerus.

- To determine the feasibility of MIPPO technique in the treatment of humeral shaft fractures (Middle and distal $1 / 3$ ) through lateral approach.

\section{METHODS}

We began our study by dissecting eight arms of four cadavers to ascertain the anatomical relationship between the radial nerve and the plate. This was a prospective study including eight patients above 20 years of age with closed fracture shaft of humerus (Middle and distal third) from 2010 to 2012.

\section{RESULTS}

- Surgical delay post injury - 11.16 days (5-12 days)

- Mean operation time - 71.33 minutes (55-85 minutes)

- Mean follow-up duration - 9.2 wks. (4-14 wks.)

- Mean healing time (when bridging callus was seen) - 8.3 wks. (6-13 wks.)

- Functional recovery was good (UCLA and MEPS score)

\section{CONCLUSION}

- MIPPO is safe and effective, alternative to open techniques.

- This is a biological fixation, which preserves the fracture haematoma and blood supply to the bone fragments and short operating time.

\section{KEYWORDS}

MIPPO, Shaft of Humerus Fracture, Anterior Approach.

HOW TO CITE THIS ARTICLE: Karunanithi S, Anbu S, Palaniappan M, et al. A study of functional, clinical and radiological outcome of fracture shaft of humerus mid and distal third managed by MIPPO technique. J. Evolution Med. Dent. Sci. 2016;5(72):5285-5291, DOI: $10.14260 /$ jemds/2016/1198

\section{INTRODUCTION}

MIPPO has been widely used to treat long bone shaft fractures in recent years, because of its technical advantages and satisfactory clinical outcomes. This method causes less soft tissue disruption and preserves the fracture haematoma and blood supply to the bone fragments. In general, there are

Financial or Other, Competing Interest: None.

Submission 22-07-2016, Peer Review 23-08-2016,

Acceptance 29-08-2016, Published 08-09-2016.

Corresponding Author:

Dr. Subramanian Karunanithi,

Plot No.54 \& 55,

Door No.6/1552, Anand Nagar,

No. 1 Tollgate, Bikshandar Koil,

Mannachanallur Taluk, Trichy-621216.

E-mail: rajijipmer@yahoo.co.in

DOI: $10.14260 /$ jemds $/ 2016 / 1198$ four conventional surgical approaches to the humeral shaft: posterior, anterolateral, anterior and anteromedial. Open plate fixation has generally used only the anterolateral and posterior approaches. The anterolateral approach is suitable for proximal and middle third fractures, whereas distal third fractures are best treated using the posterior approach. The anteromedial approach is less useful because of intervening neurovascular structures. The anterior approach is a safe and feasible way to use minimally invasive percutaneous plate osteosynthesis (MIPPO) in the treatment of humeral shaft fractures proven by Dr. T. Apivatthakakul and Colleagues. ${ }^{1}$ We undertook a study to determine the safety of MIPPO technique and also to evaluate the clinical, radiological and functional outcomes in the treatment of humeral shaft fractures (middle and distal third). 


\section{AIM OF STUDY}

The aim of the cadaveric study was to observe the anatomical relationship between the Radial Nerve and the plate during supination and pronation of forearm, so as to determine which position of forearm was safest for radial nerve in distal humerus.

The aim of the clinical study was to determine the safety of MIPPO technique and also to evaluate the clinical, radiological and functional outcomes in the treatment of humeral shaft fractures (middle and distal third).

\section{MATERIALS AND METHODS}

This study was carried out with approval of the Medical Ethics Committee of Government Kilpauk Medical College. An anatomical study was performed to evaluate the feasibility of MIPPO for the humeral shaft fractures, and to study the relationship between the radial nerve and the plate with the forearm in full pronation and in supination. The study was performed on eight arms from four fresh cadavers that were obtained from $72 \mathrm{hrs}$. to $120 \mathrm{hrs}$. after death from the Department of Anatomy and Forensic Medicine, after which we have done a prospective study of twenty cases of fracture shaft of humerus treated with minimally invasive percutaneous plate osteosynthesis from 2010 to 2012 . Clinical, radiological and functional outcomes were carried out in all 20 cases.

\section{Inclusion Criteria}

- Age above 20 years.

- Fracture shaft of humerus (middle and distal third).

- Closed displaced unstable fractures.

\section{Exclusion Criteria}

- Open fractures.

- Neurovascular injury.

- Distal humerus fracture with intra-articular extension.

- Fracture of proximal third of humerus.

- Pathological fractures.

- Skeletally immature patients.

- Patients in which time lag between injury and surgical intervention exceeded three weeks.

\section{Cadaveric Study}

The study based on cadaveric study by Apivatthakakul $\mathrm{T}$ et $\mathrm{al}^{2}$ was performed on eight arms from four fresh cadavers. Two separate incisions, one proximal and one distal, were made in each arm with the forearm in full supination. A 10holed narrow DCP was inserted into a tunnel using an anterior approach and fixed with 2 screws each on the proximal and distal humerus. The tunnel was then explored to identify the relationship between the radial nerve and the plate. No radial nerve compression or entrapment by the plate was found.

The proximal incision involves deep dissection between the lateral border of biceps muscle and medial border of deltoid muscle. The distal incision involves the retraction of the biceps muscle, medial portion of Brachialis muscle and Musculocutaneous nerve by the medial retractor. The lateral portion of Brachialis muscle served as a cushion for the radial nerve that was retracted by the lateral retractor. The tunnelling instrument was beneath the Brachialis muscle toward the proximal incision. The intimately blended fibres of the Brachialis and deltoid were incised to allow the passage of the tunnelling instrument through to the proximal incision. A longitudinal incision from the posterior part of the Acromion process to the lateral epicondyle was made to identify the axillary nerve, radial nerve and plate. Joining the MIPPO incisions and dissecting deeply to the plate in order to identify the radial nerve. The relationship between the radial nerve and the plate with the forearm in supination was 2.8 $4.6 \mathrm{~mm}(3.6 \mathrm{~mm})$. The relationship between the radial nerve and the plate with the forearm in pronation - Radial nerve moves close to the plate by $0-3 \mathrm{~mm}$. To reduce the risk of radial nerve injury, the forearm must be kept in full supination during plate insertion and excessive force should be avoided during retraction of the lateral half of the Brachialis muscle together with the radial nerve in the distal incision.

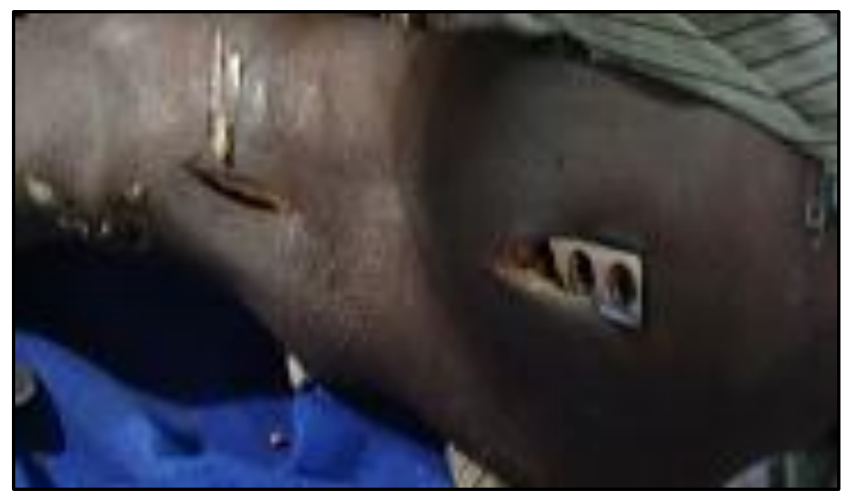

Figure 1: Cadaveric Study - Proximal and Distal Windows

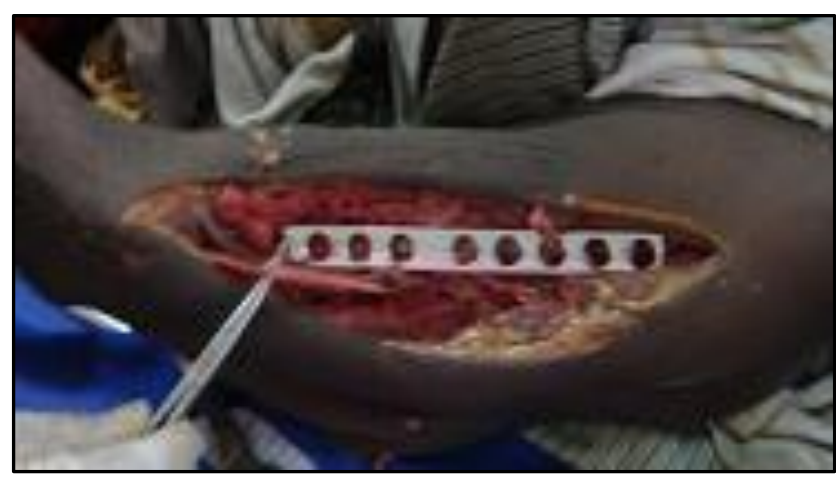

Figure 2: Cadaveric Study - Radial Nerve Position in Pronation with Plate Positioned

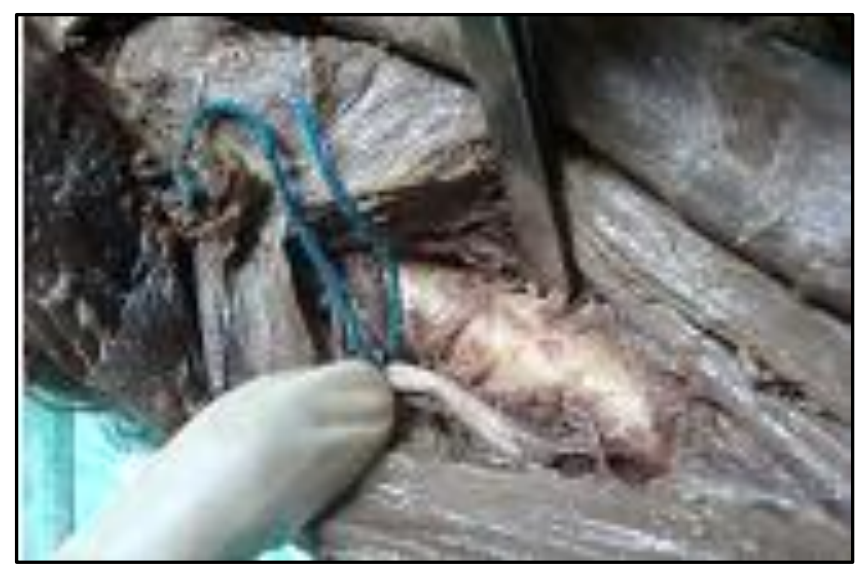

Figure 3: Cadaveric Study - Radial Nerve Moves Close to Plate while Pronation of Forearm 


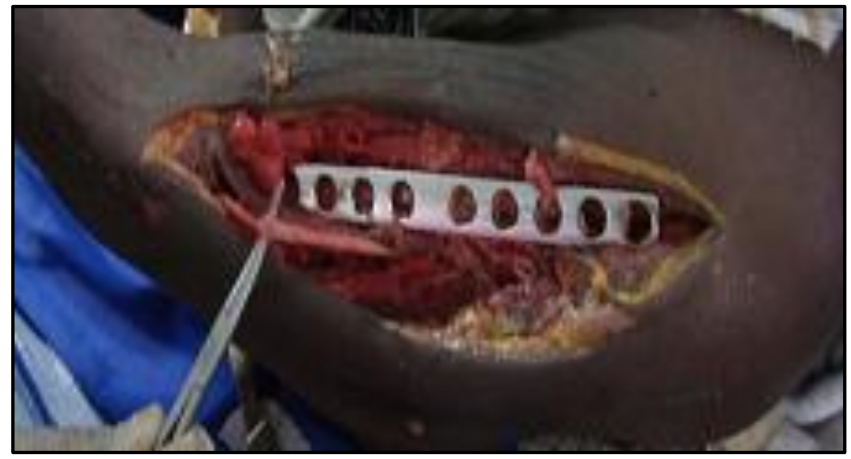

Figure 4: Cadaveric Study - Radial Nerve Position in Supination with Plate Positioned

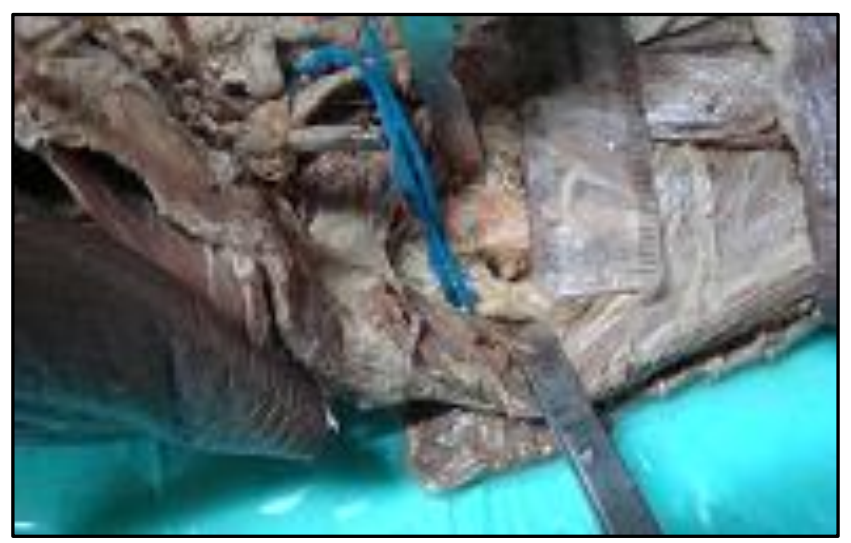

Figure 5: Cadaveric Study - Radial Nerve Moves Away from Plate while Supinating Forearm

\section{Operative Technique ${ }^{3,4}$}

The patients were placed supine on the radiolucent operating table with the injured arm in the position of 90-degree abduction and forearm in full supination to reduce the risk of radial nerve injury by maximising the distance between the radial nerve and distal portion of plate. The position of surgeon was on the lateral or cephalic side of the affected arm with the $\mathrm{C}$-arm positioned on the contralateral side to the surgeon. The first incision is $4 \mathrm{~cm}$ in length and it is made at the interval between the proximal part of biceps muscle medially and deltoid muscle laterally. Dissection is then carried down to the humerus, where the anterior border of humerus distal to the crest of greater tubercle is identified. The anterior border of humerus of the humeral shaft runs from the greater tubercle proximally and to the coronoid fossae distally. The line is almost straight, so that the straight plate can be placed on it without pre-contouring.

Distally, a $4 \mathrm{~cm}$ incision is made along the lateral border of biceps muscle approximately $1 \mathrm{~cm}$ proximal to the elbow flexion crease. The lateral quarter of the Brachialis muscle is then split longitudinally to expose the anterior cortex of the distal humerus.

A sub-muscular extraperiosteal tunnel is prepared between the Brachialis muscle and the underlying periosteum with a narrow periosteal elevator or Cobbs elevator inserted first from proximal incision distally and then from distal incision proximally. Through this tunnel a straight non-contoured long narrow $4.5 \mathrm{~mm}$ dynamic compression plate (DCP, 10 to 12 holes) or locking compression plate is inserted from proximal incision, passing over the fracture site and down to the distal incision. Special care should be taken to introduce the implant delicately in the subperiosteal region from proximal to distal in mid-shaft fractures, always with the elbow joint in semi-flexion. Soft tissue handling should be as gentle and atraumatic as possible. Retractors should be very carefully handled to avoid damage to the radial nerve by stretching or contusion. Retractors of the Hohmann type should be avoided. The implant should be accurately positioned, fixed and set on the anterior surface of the humeral shaft.

One assistant maintains the elbow in nearly $80^{\circ}$ of flexion and the forearm in supination, while keeping traction on the arm to prevent the fragments from shortening if any. A 2.0 $\mathrm{mm} \mathrm{K}$-wire is then inserted both proximally and distally through a screw hole of the plate to temporarily fix the plate. There are usually 2 screw holes exposed with each incision.

The displacement of the fractures and the length and the position of the plate are then identified under the C-arm. The proximal end of the plate is located on the anterior portion of the humerus distal to the crest of the greater tubercle. The distal end of the plate is positioned on the anterior middle line of the distal fragment just proximal to the coronoid fosse. Care is taken to not extend the distal end of the plate to the coronoid fosse; screw placement can be precarious in that location.

When the length of the humerus is approximately restored and both the ends of the plate are in the correct positions mentioned above, the proximal and distal portions of the plate are then fixed to the proximal and distal main fragments, respectively. This is accomplished by placing one screw through the incision into the screw hole not occupied by the K-wire. The screws are then inserted in neutral mode and are not tightened temporarily.

The K-wires are then removed, the apposition and the alignment of the fragments are then checked again with Carm and significant valgus or varus if any is corrected by manual manipulation. As long as a straight plate has been fixed exactly on the anterior border of the humerus which is not always easy, there will be no significant rotation or angulation.

Any significant mal-alignment is compensated by shoulder range of motion, as in the closed treatment of these fractures. The anatomical alignment is believed to be restored when the skin crease of the arm is normal in appearance and the longitudinal axes of the main fragments and the plate are parallel to each other when visualised with the C-arm. When the anatomical alignment has been achieved, the previously placed screws are then tightened definitively and further fixation of the plate is accomplished by inserting screws either percutaneously or under direct vision with at least 3 screws proximal and distal to the fracture site. The insertion of the screws can be done either through the skin incisions or additional stab incisions. The radial nerve is not exposed during this procedure.

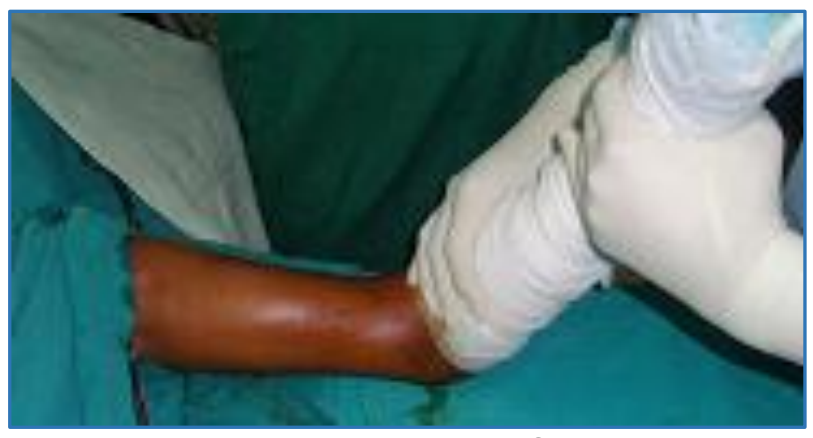

Figure 6: Position of Arm 


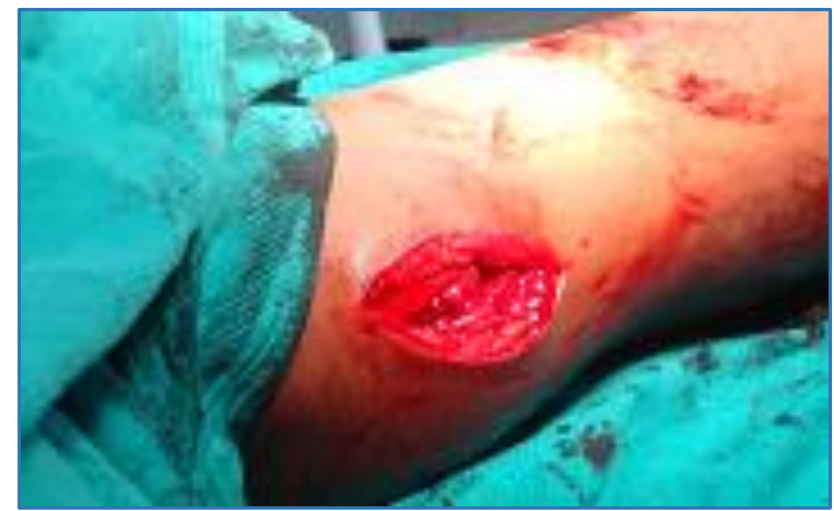

Figure 7: Proximal Window

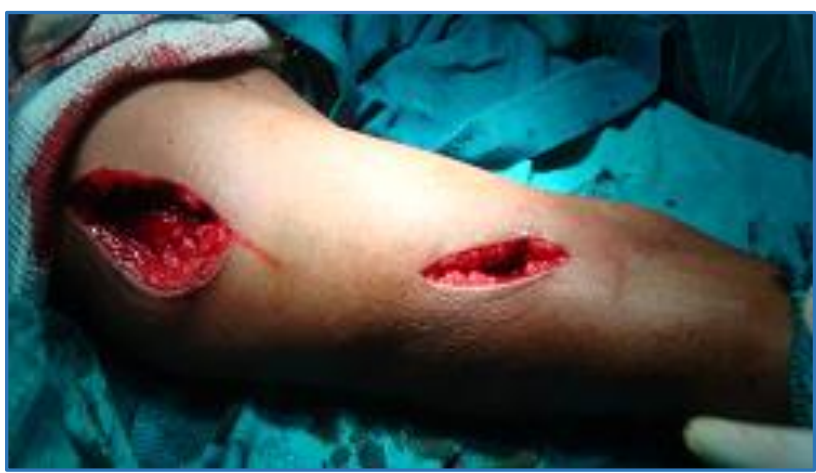

Figure 8: Both Proximal and Distal Windows

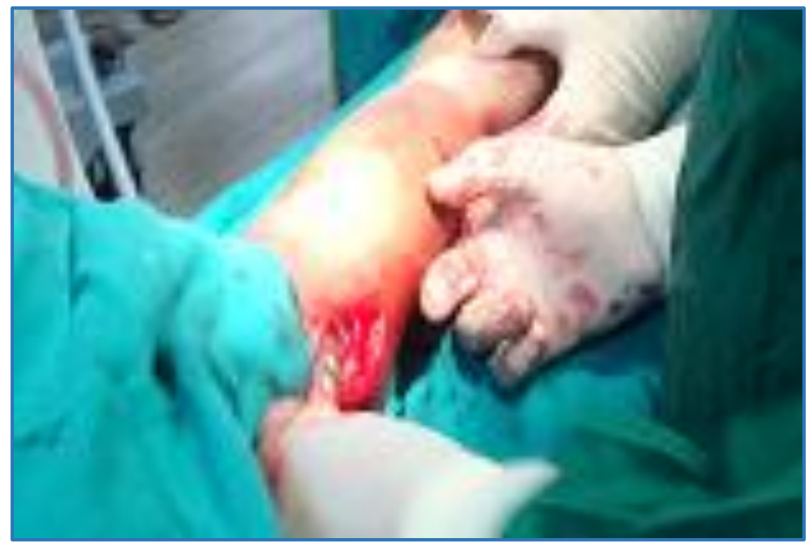

Figure 9: Extraperiosteal Tunnel Creation

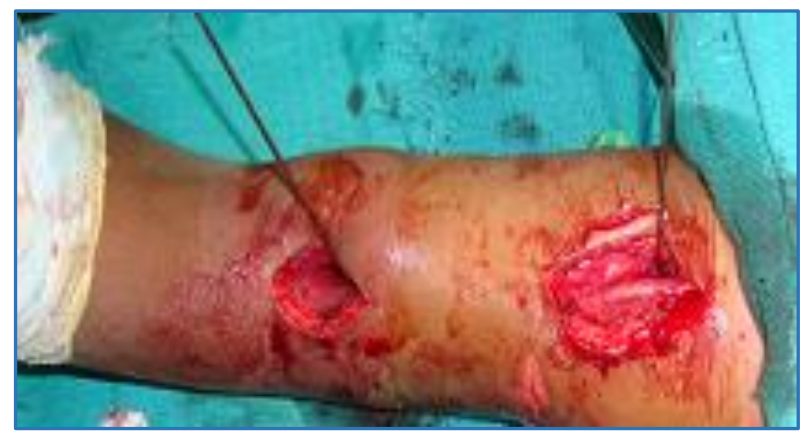

Figure 10: Plate Provisionally Fixed with K Wires

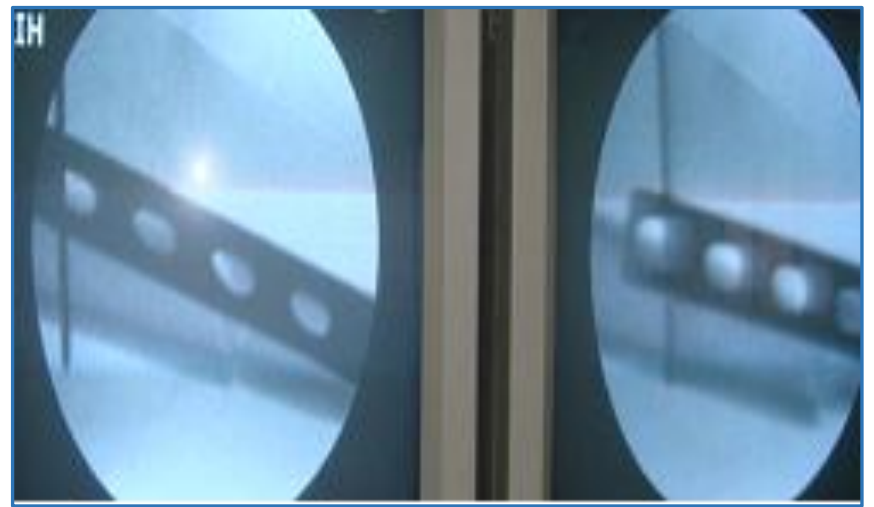

Figure 11: C - Arm Images

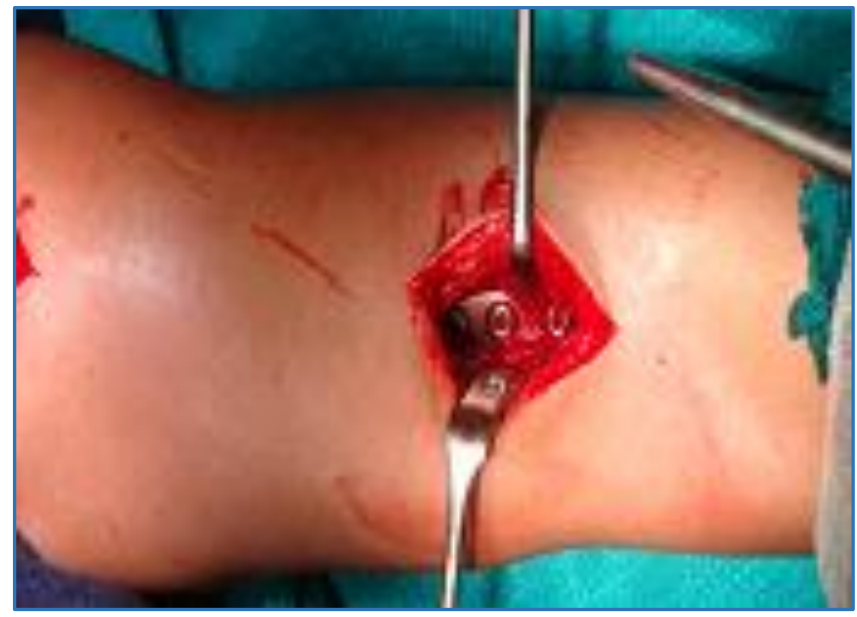

Figure 12: After Plate Fixation

Post-Operative Protocol ${ }^{3,4}$

- Passive motion of shoulder and elbow in first week.

- Active motion from second week.

- Wound inspection on day-2.

- Suture removal on $12^{\text {th }}$ day.

- Progressive increase in weight lifting.

- Serial X-rays in monthly interval to look for union.

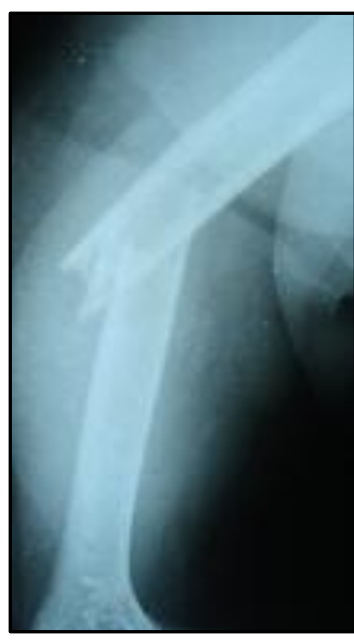

Figure 13: Pre-Op X-Ray-AP

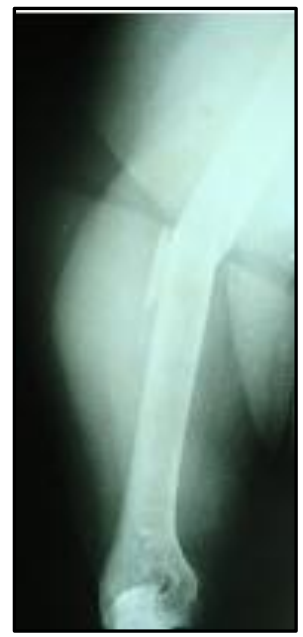

Figure 14: Lateral View 


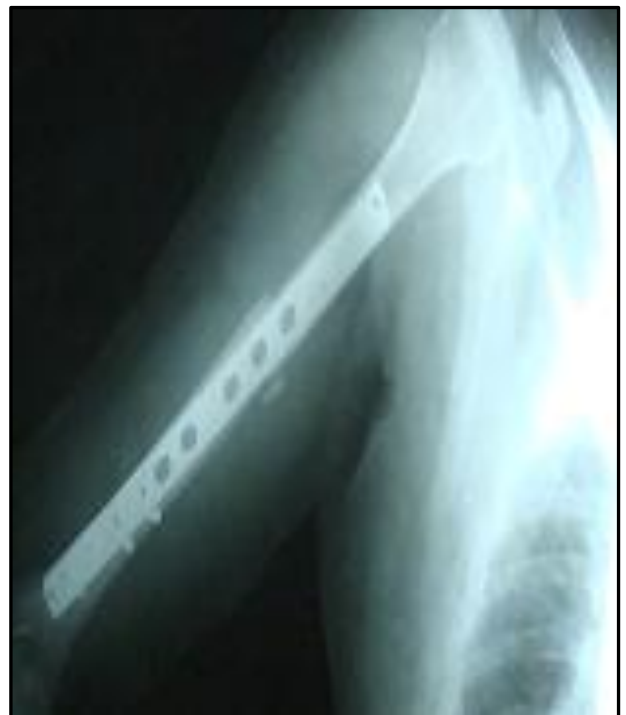

Figure 15: Immed Post-Op X-Ray-AP

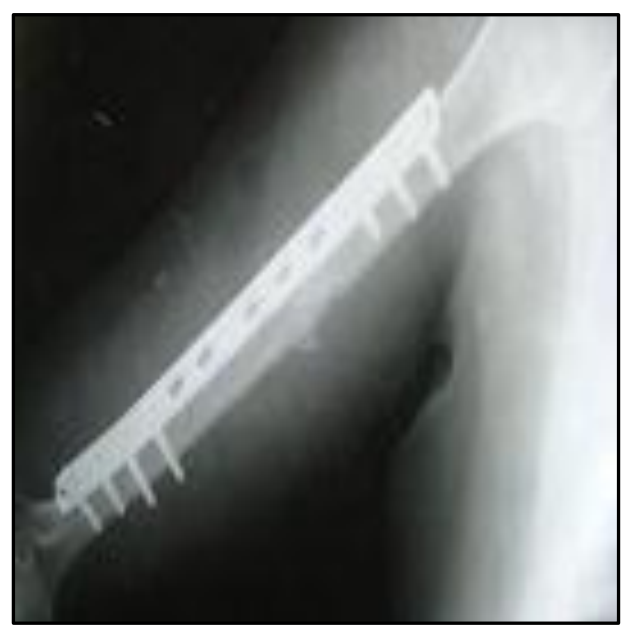

Figure 16: Lateral View

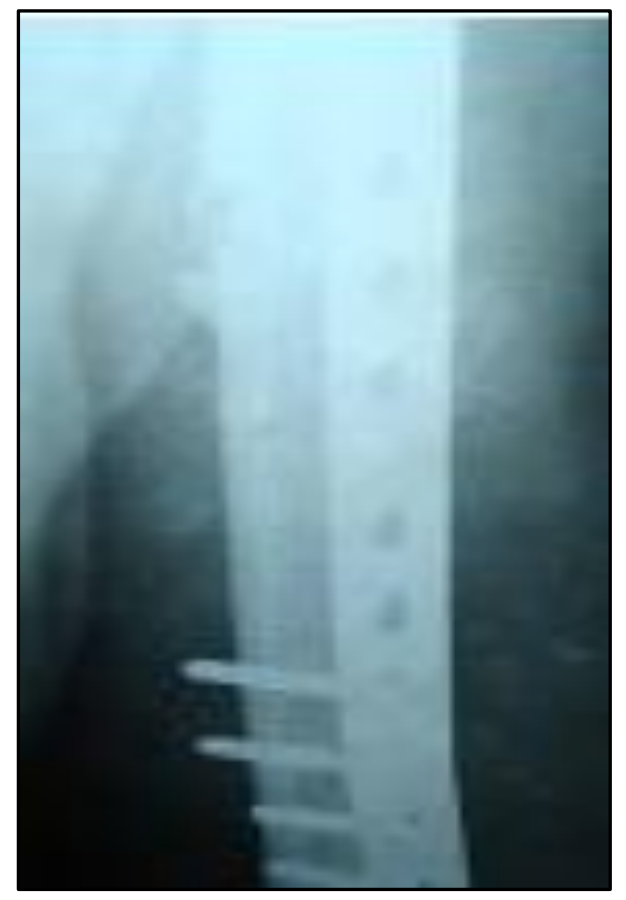

Figure 17: 6 Weeks Post-Op

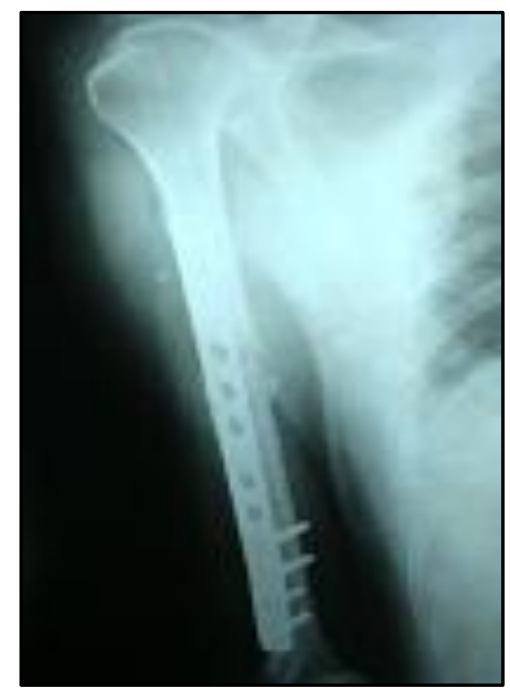

Figure 18: 12 Weeks Post-Op - AP

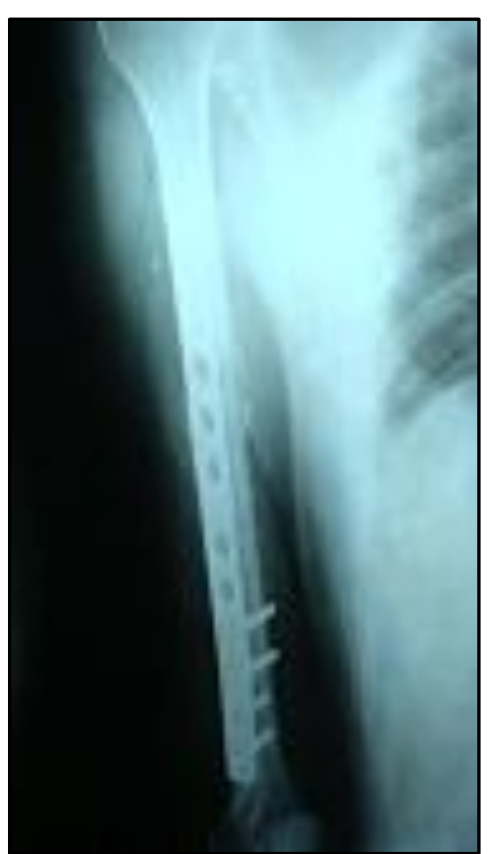

Figure 19: Lateral

\section{RESULTS}

The data collected from the patients - sex, injured side, rate of associated injuries, rate of postoperative complications and fracture characteristics were analysed. Statistical analysis was performed using SPSS software, version 13.0 (SPSS, Inc., Chicago, IL, USA).

\section{Cadaveric Study}

The plates were inserted extra-periosteally beneath the Brachialis in all 8 specimens. The plate was laid on the anterior surface of the humerus with a thin layer of muscle against it. There was no radial nerve entrapment by the plate in any case. The radial nerve ran close to the bone in the spiral groove on the posterior surface of the middle third of the humerus. After piercing the lateral intermuscular septum, it comes to the anterior compartment. The radial nerve was separated by a thin layer of muscle from the lateral cortex of the distal humerus. The Musculocutaneous nerve ran between the biceps and Brachialis muscles and could be protected by the medial retractor. 


\section{Clinical Study}

Twenty patients were treated by applying the MIPPO technique. All patients had intact radial nerve function before the operation. All cases were operated on using a double small approach by the MIPPO technique under fluoroscopic control, as described in the cadaveric study. The plates were fixed with three to four screws in both proximal and distal fragments. The wound was closed without drains or external immobilisation. The patients were encouraged to perform passive motion of the shoulder and elbow during the first postoperative week. Active motion was carried out from the second week after the operation without a cast or brace.

The mean age of the patients was 41.30 years (range 21 65 yrs.). The average operating time was 100.50 minutes (range 70 - 140 mins.). The average time interval between injury and surgery was 7.25 days (range 3-15 days). The average duration of follow-up was 36.50 weeks (range 24-72 weeks). Two patients had radial nerve dysfunction after the operation. We re-explored the Radial nerve in one patient and found that the radial nerve had not been trapped under the plate and its damage was due to dragging and contusion. The patient's radial nerve function fully recovered 6 months after the second operation.

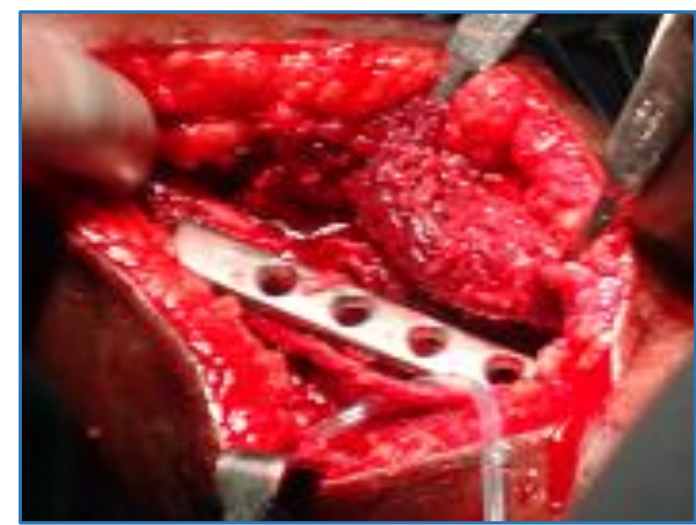

Figure 20: Nerve Exploration

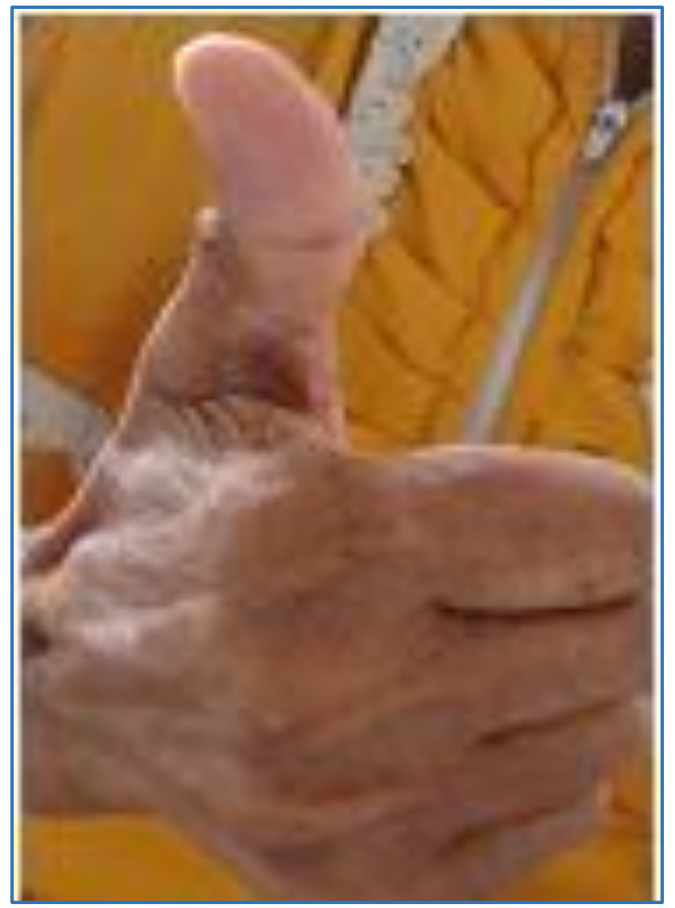

Figure 21: Radial Nerve Function Recovered
Another patient's radial nerve function recovered after 12 weeks without any secondary procedure.

Union was achieved in all patients without bone grafting. One patient had delayed union for which we gave brace and then it went on to unite after 32 weeks. All fractures united with a mean healing time of 17.84 weeks (range 12 - 32 weeks).

The UCLA scoring system showed excellent results in nine cases (45\%) and good results in seven cases (35\%) and fair in 4 cases $(20 \%)$.

Eighteen patients (90\%) had excellent results; two patients $(10 \%)$ had good results of their elbow function when assessed with the Mayo elbow performance scoring system. No cases of superficial or deep infection and implant failures were noted in our study.

At their final follow-up, all the twenty patients had an antero-posterior and lateral radiographs of the humerus taken to measure the humeral axial alignment. Normal alignment was seen in nine (45\%) cases, whereas varus angulation was found in seven $(35 \%)$ cases and valgus deformity in four $(20 \%)$ cases.

\section{DISCUSSION}

Dr. T. Apivatthakakul ${ }^{1}$ and Colleagues have verified that the MIPPO technique can be safely and efficiently applied in the treatment of humeral shaft fractures through the anterior approach. Apivatthakakul $\mathrm{T}$ et $\mathrm{al}^{1}$ performed on ten arms from five fresh cadavers. No radial nerve compression or entrapment by the plate was found. The distance measured from the closest part of the plate to the radial nerve was 2.0 $4.9 \mathrm{~mm}$ (average $3.2 \mathrm{~mm}$ ). When the forearm was pronated, the radial nerve moved closer to the plate by a distance of $0-3$ $\mathrm{mm}$. The results of this study showed that it is possible to treat humeral shaft fractures by the MIPPO method using an anterior approach.

The results from the cadaveric study by Fang Ji et $\mathrm{al}^{4}$ have shown that it is possible, safe and convenient to perform the MIPPO technique for the humeral shaft fractures (mid and distal third). The closest distance measured between the lateral border of the plate and the radial nerve in full supination of the forearm was $2.5-5.3 \mathrm{~mm}$ (average $3.7 \mathrm{~mm}$ ). When the forearm was pronated, the radial nerve was noted to move medially closer to the distal end of the plate. The radial nerve moved to touch the plate in four specimens, while in two specimens there were distances of 1.3 and 1.5 $\mathrm{mm}$.

In our cadaveric study, we found that the closest distance measured between the lateral border of the plate and the radial nerve in full supination of the forearm was $2.8-4.6$ $\mathrm{mm}$ (average $3.6 \mathrm{~mm}$ ). When the forearm was pronated, the radial nerve was noted to move medially closer to the distal end of the plate by a distance of 0-3 mm.

In our clinical study of 20 patients, all had fracture union with a mean healing time of 17.84 weeks (range 12 - 32 weeks), comparable to Zhiquan A et $\mathrm{al}^{3}$ - 16.2 weeks (range 12 to 32 weeks) and Sang-Jin Shin et al ${ }^{5}-18.4$ weeks.

The mean operating time in our study was 100.50 minutes (range $70-140$ mins.), whereas in Zhiquan A et $\mathrm{al}^{3}$, it was 113.8 minutes (70-160 mins.), and Fang Ji et al ${ }^{4}$ it was only 48 minutes (35-90 mins.). 
In our study we encountered 2 cases of radial nerve dysfunction post-operatively, which recovered after 6-12 weeks in one case and 6 months in another case, whereas Zhiquan A et $\mathrm{al}^{3}$ and Apivatthakakkul et $\mathrm{al}^{1,2}$ reported no cases of radial nerve dysfunction after surgery. However, Fang Ji et $\mathrm{al}^{4}$ in his study reported one patient with radial nerve dysfunction which recovered five months after second surgery and Pospula et al ${ }^{6}$ reported one patient with transient radial nerve palsy. Livani et al ${ }^{7}$ reported good results in 35 cases of mid-distal humeral shaft fractures without iatrogenic radial nerve lesions. In Sang-Jin Shins et $\mathrm{l}^{5}$ study one patient had radial nerve palsy.

In the reviewed literature, there were no reports of nonunion after MIPPO technique. However, in our series, one patient had delayed union for which we gave brace and then it went on to unite after 32 weeks.

In Zhiquan $\mathrm{A}$ et $\mathrm{al}^{3}$ study, UCLA scoring system rated seven patients (63.8\%) as excellent results and six (46.2\%) as good results. In Sang-Jin Shin et al study, 5 (31 patients)-9 were excellent and 3 were good for the proximal humeral fractures, excellent results in 15 cases and good in 4 cases for middle and distal third fractures. Whereas, in our study the UCLA scoring system showed excellent results in nine cases $(45 \%)$ and good results in seven cases (35\%) and fair in 4 cases $(20 \%)$.

In Zhiquan $\mathrm{A}$ et $\mathrm{al}^{3}$ MEPS score was excellent in all cases, whereas in our series eighteen patients $(90 \%)$ had excellent results, two patients (10\%) had good results.

In comparison with open reduction and internal fixation using a plate, one of the major theoretical advantages of MIPPO with an anteriorly placed plate to treat mid-distal humeral shaft fractures is less surgical trauma to the soft tissue around the fracture site. The periosteal circulation around the fracture fragments is minimally disrupted and thus bone union is promoted and complications such as nonunion are decreased.

\section{CONCLUSION8,9,10}

At the end of this study we can emphasise the advantages of this technique regarding safety and convenience, without requiring special tools and demanding implants or excessive radiographic control. The plate stability allows a fast rehabilitation with superior functional results comparing with the conservative techniques.

Mid-distal third humeral shaft fractures could be effectively treated with the MIPPO technique with advantages of shorter fracture union time and lower incidence of iatrogenic radial nerve palsies, but with similar functional outcomes to the conventional open plating technique.

\section{REFERENCES}

1. Apivatthakakul T, Phornphutkul C, Laohapoonrungsee A, et al. Less invasive plate osteosynthesis in humeral shaft fractures. Operative Orthopadie und Traumatologie 2009;21(6):602-13.

2. Apivatthakakul T, Arpornchayanon O, Bavornratanavech S. Minimally invasive plate osteosynthesis (MIPO) of the humeral shaft fracture. Is it possible? A cadaveric study and preliminary report. Injury 2005;36(4):530-8.

3. Zhiquan A, Bingfang $\mathrm{Z}$, Yeming $\mathrm{W}$, et al. Minimally invasive plating osteosynthesis (MIPO) of middle and distal third humeral shaft fractures. J Orthop Trauma 2007; 21(9):628-33.

4. Ji F, Tong $\mathrm{D}$, Tang $\mathrm{H}$, et al. Minimally invasive percutaneous plate osteosynthesis (MIPPO) technique applied in the treatment of humeral shaft distal fractures through a lateral approach. Int Orthop (SICOT) 2009;33(2):543-7.

5. Sang-Jin S, Hoon-Sang S, Nam-Hoon D, et al. Minimally invasive plate osteosynthesis of proximal, middle and distal humerus fractures. J Korean Orthop Assoc 2010; 45(6):448-55.

6. Pospula W, Noor AT. Percutaneous fixation of comminuted fractures of the humerus: initial experience at al razihospital, Kuwait. Med Princ Pract 2006;15 (6):423-6.

7. Livani B, Belangero $W$, Andrade $K$, et al. Is MIPO in humeral shaft fractures really safe? Postoperative ultrasonographic evaluation. Int Orthop 2009;33(6) :1719-23.

8. Perren SM. Evolution of the internal fixation of long bone fractures. The scientific basis of biological internal fixation: choosing a new balance between stability and biology. J Bone Joint Surg Br 2002;84(8):1093-110.

9. Perren SM. The technology of minimally invasive percutaneous osteosynthesis (MIPO). Injury 2002;33 (Suppl 1):6-7.

10. Chao-Lai J, Wei J, Xiao-Xing X. A self designed anatomical anterior locking plate for minimally invasive treatment of mid-distal humeral fractures. Chinese Journal of Orthop Trauma 2011;13(4):362-5. 